\title{
VASSILIEV INVARIANTS AS POLYNOMIALS
}

\author{
SIMON WILLERTON \\ Department of Mathematics and Statistics, University of Edinburgh \\ James Clerk Maxwell Building, King's Buildings, Edinburgh, EH9 3JZ, Scotland \\ E-mail: simon@maths.ed.ac.uk
}

\begin{abstract}
Three results are shown which demonstrate how Vassiliev invariants behave like polynomials.
\end{abstract}

0. Introduction. The purpose of this note is to show how the phrase "Vassiliev invariants are like polynomials" can be a useful working paradigm. This has been illustrated in other talks at this conference: Deguchi used Vassiliev invariants because they are computable in polynomial time (see below) and Burri [4] demonstrated that, for a fixed shadow, Vassiliev invariants are polynomial functions in the gleams.

Here three other results are presented which reflect the motto in some manner. They are as follows: the fact that Vassiliev invariants form a filtered algebra; Dean and Trapp's criterion for an invariant to be of finite type; and a partial solution to the problem of integrating a weight system.

Two results with a polynomial flavour which don't quite fit into the framework of this note are that the value of an invariant of degree $n$ on a knot with $c$ crossings is bounded by a polynomial of degree $n$ in $c$ and that the value is calculable in similarly polynomial time. These follow from Stanford's algorithm [11] for calculating Vassiliev invariants and were also proved by Bar-Natan [2].

For simplicity all invariants will take values in $\mathbf{Q}$.

Recall Birman and Lin's axiomatic reformulation of Vassiliev invariants [3].

Definition. For $i \in\{0,1,2, \ldots\}$, let $X_{i}$ be the set of (oriented) "knots with $i$ double points" or " $i$-singular knots", i.e. maps $S^{1} \rightarrow \mathbf{R}^{3}$ such that the only possible singularities are $i$ transversal self-intersections, considered up to rigid vertex isotopy.

For instance, $X_{0}$ is just the set of usual knots up to ambient isotopy. Suggestive language will be used for the extension of a knot invariant to knots with double points:

1991 Mathematics Subject Classification: Primary 57M25.

The paper is in final form and no version of it will be published elsewhere. 
Definition. Given a knot invariant $V: X_{0} \rightarrow \mathbf{Q}$, define its ith derivative, $V^{(i)}: X_{i} \rightarrow$ $\mathbf{Q}$, inductively via the so-called Vassiliev skein relation:

$$
V^{(0)}=V ; \quad V^{(i)}(\succ)=V^{(i-1)}(\nearrow)-V^{(i-1)}(\nearrow) .
$$

Remark. (i) It is straight-forward to check that this definition is independent of the order in which the double points are resolved.

(ii) The "Vassiliev-space" way of thinking of this is that the space of knots with double points is stratified, each stratum being co-oriented in the one above, so the invariant can be naturally extended from "chambers" to "walls" (see Fig. 1) by measuring the "jump" in the invariant when passing through the wall in a positive direction.
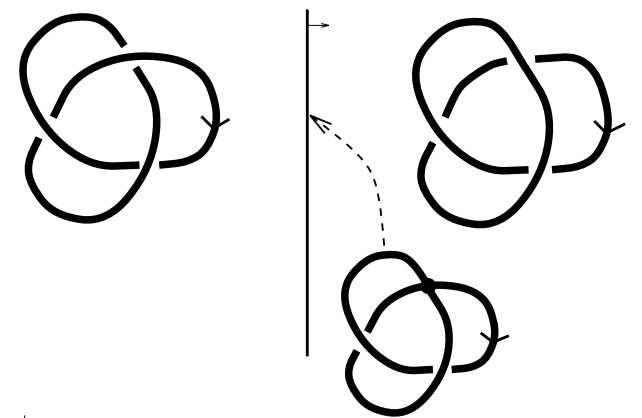

Fig. 1. The pictured singular knot lies on the co-oriented wall between the chambers containing the unknot and the negative trefoil.

Definition. A knot invariant $V: X_{0} \rightarrow \mathbf{Q}$ is said to be Vassiliev, of finite type, or of type $n$, if there exists $n \in \mathbf{N}$ such that $V^{(n+1)}=0$. (These invariants clearly form a vector space.)

The key idea for this note, observed in [1], is that this condition is analogous to the vanishing derivative condition for polynomials.

1. A Leibniz-like theorem. Two knot invariants, $V$ and $W$, can be multiplied pointwise, i.e.

$$
(V . W)(K):=V(K) W(K) .
$$

One can perhaps see from Vassiliev's spectral sequence approach [13] that this makes the vector space of Vassiliev invariants into a filtered algebra, but how can one see it from the axiomatic description? For products of polynomials one could use the Leibniz theorem:

TheOREM 1. If $f, g: \mathbf{R}^{m} \rightarrow \mathbf{R}$ are functions, then

$$
\frac{\partial^{i}(f g)}{\partial x_{1} \ldots \partial x_{i}}=\sum_{J \subseteq\{1, \ldots, i\}} \frac{\partial^{|J|} f}{\partial x_{J}} \cdot \frac{\partial^{|\bar{J}|} g}{\partial x_{\bar{J}}},
$$

where $\bar{J}=\{1, \ldots, i\} \backslash J$ and the multi-index notation means that for $J=\left\{j_{1}, \ldots, j_{l}\right\}$ one has $\partial x_{J}=\partial x_{j_{1}} \partial x_{j_{2}} \ldots \partial x_{j_{l}}$.

In fact an analogous theorem holds for knot invariants. First, introduce a little bit of extra notation: for $P$ an invariant of knots with $l$ double points, and $k$ a knot with $i$ double points, let $I=\{1, \ldots, i\}$ be a labeling set for the double points, then for $J \subset I,|J|=l$ 
let $P\left(k_{J}\right)$ be the average of $P$ evaluated on $2^{i-l}$ knots with $l$ double points obtained by the different resolutions of the double points not in $J$. This corresponds to the idea of $\mathcal{V} \mapsto \frac{1}{2}(Y+X)$ for the double points not in $J$.

Theorem 2 [14]. If $V, W: X_{0} \rightarrow \mathbf{Q}$ are knot invariants, and $k \in X_{i}$ is a knot with $i$ double points, then

$$
(V . W)^{(i)}(k)=\sum_{J \subseteq\{1, \ldots, i\}} V^{(|J|)}\left(k_{J}\right) \cdot W^{(|\bar{J}|)}\left(k_{\bar{J}}\right) .
$$

An immediate corollary of this is that the space of finite type invariants is a filtered algebra or in other words:

Corollary 3. If $V, W: X_{0} \rightarrow \mathbf{Q}$ are type $m$ and type $n$ knot invariants respectively then $V . W$ is of type $n+m$.

Proof. In the expression for $(V . W)^{(n+m+1)}$, in each summand either $|J|>m$ or $|\bar{J}|>n$, hence either $V^{(|J|)}=0$ or $W^{(|\bar{J}|)}=0$. Whence $(V . W)^{(n+m+1)}=0$.

See also $[7,8]$ for other approaches to this corollary.

2. A Newton-like polynomial criterion. Now move from a Leibniz-type formula to a criterion of Dean and Trapp, analogous to one attributed to Newton.

Definition. A family of knots $\left\{K_{i}\right\}_{i \in \mathbf{Z}} \subset X_{0}$ is a twist sequence (see Fig. 2) if the following hold:

(i) $K_{0}$ can be represented by a diagram in which there is a disc in $\mathbf{R}^{2}$ which intersects the diagram as two parallel lines;

(ii) $K_{i}$ is obtained, for $i>0$ (respectively $i<0$ ), by replacing the two lines by $i$ positive full twists (respectively $-i$ negative full twists).

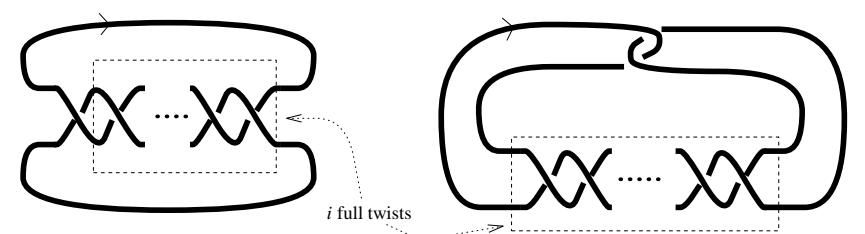

Fig. 2. Two twist sequences: the $(2,2 i+1)$ torus knots, and the twisted Whitehead doubles of the unknot.

Newton had a criterion for polynomial functions, let $f: \mathbf{Z} \rightarrow \mathbf{R}$ be a function then define $\Delta^{0} f=f$ and inductively $\Delta^{n} f(x)=\Delta^{n-1} f(x+1)-\Delta^{n-1} f(x)$.

ThEOREM 4 (Newton). The function $f$ is a polynomial of degree less than or equal to $i$ if and only if $\Delta^{i+1} f=0$.

Given a knot invariant $V$ and a twist sequence $\left\{K_{i}\right\}_{i \in \mathbf{Z}}$ define the function $\tilde{V}_{K}: \mathbf{Z} \rightarrow \mathbf{Q}$ by $i \mapsto V\left(K_{i}\right)$. Newton's criterion translates, via the Vassiliev skein relation to the following: 
TheOREm $5[5,12]$. If $V$ is of type $n$ then $\tilde{V}_{K}$ is a polynomial of degree less than or equal to $n$.

This can now be used to prove that various classical invariants are not of finite type.

Corollary 6 [5, 12]. Unknotting number, signature, genus, bridge number, and crossing number are not of finite type.

As an example, consider the unknotting number, $U$, evaluated on the twisted Whitehead doubles of the unknot (see Fig. 2),

$$
U(W h(i))= \begin{cases}0 & i=0 \\ 1 & \text { otherwise }\end{cases}
$$

This is clearly not a polynomial in $i$ of any degree and so $U$ is not of finite type. (This example is essentially the same as in [3].)

Remark. Stanford [10] has generalized these ideas to "tangle maps". In this case $K_{0}$ is a knot which has a diagram which intersects some disc as $l$ parallel strands. He shows that if $\left\{T_{1}, \ldots, T_{j}\right\}$ is a set of commuting, pure $l$-tangles, and $K\left(i_{1}, \ldots, i_{j}\right)$ is the knot obtained from $K_{0}$ by putting the tangle $T_{1}^{i_{1}} T_{2}^{i_{2}} \ldots T_{j}^{i_{j}}$ where the parallel strands are, and if $V$ is a type $n$ knot invariant, then $V\left(K\left(i_{1}, \ldots, i_{j}\right)\right)$ is a polynomial in the $\left\{i_{s}\right\}_{s=1}^{j}$ of degree at most $n$.

3. Integration. An important notion is that of anti-differentiation, or integration - going from an invariant of knots with $n$ double points to an invariant of knots with $n-1$ double points - if an invariant is defined on the walls can it be extended to the chambers? So, say that $R: X_{n-1} \rightarrow \mathbf{Q}$ is a first integral of $P: X_{n} \rightarrow \mathbf{Q}$ if

$$
P(\cdot)=R(Y)-R(X) \text {. }
$$

One would like to know when given a $P$ one can find such an $R$, i.e. when $P$ can be integrated; and further when $P$ can be repeatedly integrated to an actual knot invariant. From Stanford we have the following criterion.

Theorem 7 [9]. $P: X_{n} \rightarrow \mathbf{Q}$ has a first integral if and only if it satisfies the following:

(i) topological 1 term: $P(\chi)=0$;

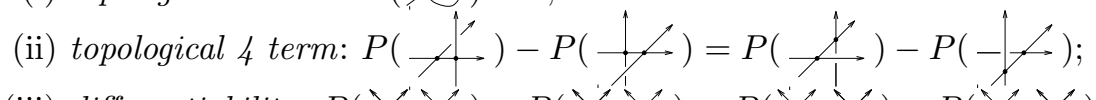

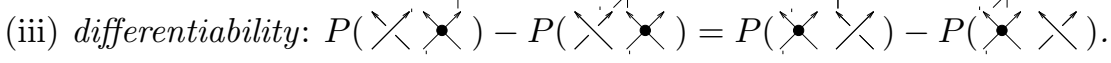

So say that an invariant is integrable if it satisfies these hypotheses. Unfortunately this theorem is inadequate in at least the following two senses: it says nothing about the possibility of integrating $R$; and it is purely an existence theorem. In fact, if $R$ exists then it is only defined up to "constants of integration"; in the case that $R$ is itself integrable, these constants are known as weight systems. (In other words, if $R$ is an integrable first integral of $P$, then $S$ is also an integrable first integral of $P$ precisely when $R-S$ is a weight system.) 


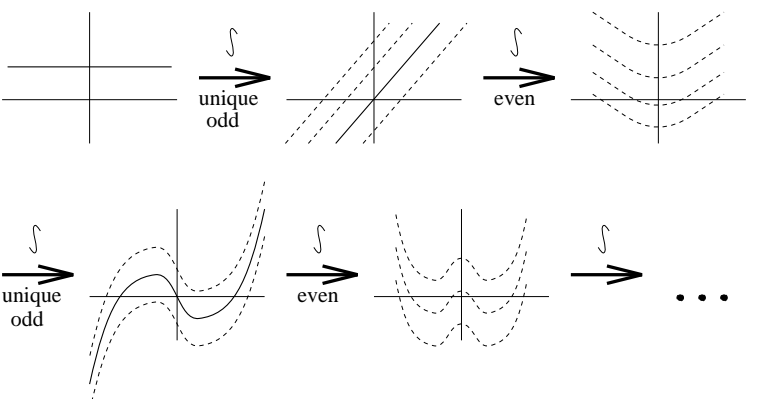

Fig. 3. Semi-systematic integration of even and odd polynomials.

3.1. Weight systems. When a degree $n$ polynomial is differentiated $n$ times, the result is a constant function. Analogously we look at the $n$th derivatives of type $n$ Vassiliev invariants.

Definition. A weight system of type $n$ is an integrable invariant of knots with $n$ double points, $W: X_{n} \rightarrow \mathbf{Q}$, which differentiates to zero $\left(W^{\prime}=0\right)$.

So if $V: X_{0} \rightarrow \mathbf{Q}$ is a type $n$ knot invariant then $V^{(n)}$ is a weight system.

FACT 1. If $W$ is a weight system, then $W$ is invariant under crossing changes.

This is clear as $W(Y)-W(X)=W^{\prime}(\succ)=0$. This fact leads to the combinatorial description of weight systems in terms of chord diagrams: this is not particularly relevant here and the reader is referred to [1] for details.

One goal is to integrate a given weight system all the way to a knot invariant. From the Kontsevich integral (see [1]) this can certainly be done over $\mathbf{Q}$ but the Kontsevich integral is neither constructive nor described in this axiomatic framework. Perhaps the polynomial analogy can help with integration.

3.2. Introducing symmetry. For a singular knot, $k$, let $\bar{k}$ be its mirror image (see Fig. 4).

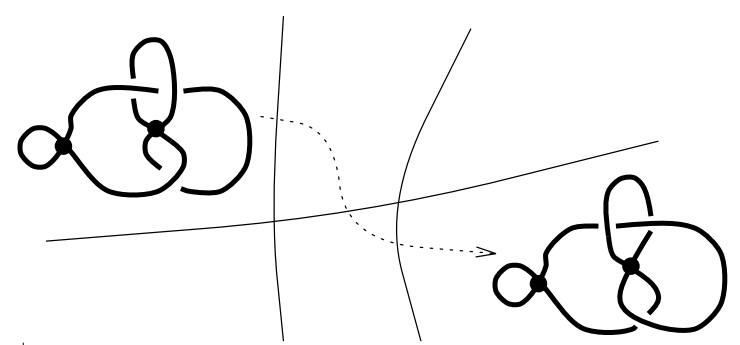

Fig. 4. A path in knot space from a singular knot to its mirror image: each passage through a wall corresponds to a change of one of the crossings in the projection.

ObSERVATION 1. (i) [6, (implicit)] If $W$ is a weight system of type $n$ then $W(k)=$ $W(\bar{k})$ for all $k \in X_{n}$.

(ii) [13, Theorem V.5.2] If $V$ is a type $n$ invariant then one can add an invariant, $V_{1}$, of lower order so that $\left(V+V_{1}\right)(k)=(-1)^{n}\left(V+V_{1}\right)(\bar{k})$ for all $k \in X_{n}$. 
(i) is clear from the fact that given a projection of $k$ one can obtain the mirror image by switching each of the crossings in turn and applying Fact 1. (ii) was obtained by Vassiliev by considering the action of taking the mirror image on his spectral sequence.

These observations should be reminiscent of the following for polynomials:

Observation 2. (i) If $f: \mathbf{R} \rightarrow \mathbf{R}$ is a constant function then $f(x)=f(-x)$ for all $x \in \mathbf{R}$.

(ii) If $g: \mathbf{R} \rightarrow \mathbf{R}$ is a degree $n$ polynomial then one can add a polynomial, $g_{1}$, of lower degree so that $\left(g+g_{1}\right)(x)=(-1)^{n}\left(g+g_{1}\right)(-x)$ for all $x \in \mathbf{R}$.

In the case of polynomials there is a semi-systematic way of integrating from a constant function to a polynomial which is either even or odd depending on the parity of the degree. This is done as follows. A constant function is even and integrates uniquely to an odd function, this resulting function will integrate - regardless of constants of integration to an even function. This even function integrates to a unique odd function, and so on (see Fig. 3). It is in this sense that half of the constants of integration can be set.

At this point, the reader will not be surprised to discover that an analogous procedure can be carried out for knot invariants. Call an invariant $P: X_{n} \rightarrow \mathbf{Q}$ even if for all $k \in X_{n}$ $P(k)=P(\bar{k})$, and odd if $P(k)=-P(\bar{k})$.

TheOREm 8 [15]. (i) If $P: X_{n} \rightarrow \mathbf{Q}$ is an even integrable invariant of knots with $n$ double points, then $P$ has a unique odd first integral and this is also integrable.

(ii) Every first integral of an odd invariant is even.

In fact the situation is slightly better because there is actually a combinatorial formula for the odd first integral, $R$, of an even invariant, $P$. A path in knot-space between an $(n-1)$-singular knot $k$ and its mirror image $\bar{k}$ can be obtained from a diagram by just switching each of the crossings, one after another (see Fig. 4). The difference between $R(k)$ and $R(\bar{k})$ is just the sum of the jumps when each of the crossing changes occurs. This is the sum (with suitable signs) of $P$ evaluated on the $n$-singular knots obtained by smashing each of the crossings in turn. However $R$ is odd, so $R(k)=-R(\bar{k})$ and hence $R(k)$ is half of this sum. (An explicit formula for this can be found in [15].)

All that is required then for a canonical integration from weight system to genuine knot invariant, is a canonical way of integrating even integrable invariants. Polynomial intuition has not yet been able to offer any help with this problem.

\section{References}

[1] D. B ar - Nata n, On the Vassiliev knot invariants, Topology 34 (1995) no. 2, 423-472.

[2] - Polynomial invariants are polynomial, Mathematical Research Letters, 2 (1995) 239246.

[3] J. Birman and X. S. Lin, Knot polynomials and Vassiliev's invariants, Invent. Math. 111 (1993), 225-270.

[4] U. B u r r i, For a fixed Turaev shadow all "Jones-Vassiliev" invariants depend polynomially on the gleams, University of Basel preprint, March 1995. 
[5] J. Dean, Many classical knot invariants are not Vassiliev invariants, J. Knot Theory Ramifications, 3 (1994) 7-9.

[6] M. Domerg u e and P. D on at o, Integrating a weight system of order $n$ to an invariant of $(n-1)$-singular knots, J. Knot Theory Ramifications, 5 (1996) 23-35.

[7] M. Gussarov, On n-equivalence of knots and invariants of finite degree, in Topology of manifolds and varieties (O. Viro, editor), Amer. Math. Soc., Providence 1994, 173-192.

[8] J. H. P rzyty cki, Vassiliev-Gusarov skein modules of 3-manifolds and criteria for periodicity of knots, Proceedings of low-dimensional topology, May 18-23 1992, International Press, Cambridge MA, 1994.

[9] T. Stanford, Finite-type invariants of knots, links, and graphs, Topology 35 (1996) 1027-1050.

[10] - , The functoriality of Vassiliev-type invariants of links, braids, and knotted graphs, J. Knot Theory Ramifications, 3 (1994) 247-262.

[11] - Computing Vassiliev's invariants, University of California at Berkeley preprint, December 1995.

[12] R. Trapp, Twist sequences and Vassiliev invariants, J. Knot Theory Ramifications., 3 (1994) 391-405.

[13] V. A. Vassiliev, Complements of discriminants of smooth maps: topology and applications, Trans. of Math. Mono. 98, Amer. Math. Soc., Providence, 1992.

[14] S. Willerton, Vassiliev knot invariants and the Hopf algebra of chord diagrams, Math. Proc. Camb. Phil. Soc., 119 (1996) 55-65.

[15] - , A combinatorial half-integration from weight system to Vassiliev knot invariant, J. Knot Theory Ramifications, to appear. 